\title{
Resistive switching and charge transport mechanisms in ITO/ZnO/p-Si devices
}

\author{
O. Blázquez, ${ }^{1,2, a)}$ J. L. Frieiro, ${ }^{1,2}$ J. López-Vidrier, ${ }^{3}$ C. Guillaume, ${ }^{4}$ X. Portier ${ }^{4}$ C. Labbé, ${ }^{4}$ \\ P. Sanchis, ${ }^{5}$ S. Hernández,,$^{1,2}$ and B. Garrido ${ }^{1,2}$ \\ ${ }^{1}$ MIND, Department of Electronics and Biomedical Engineering, Universitat de Barcelona, Martí $i$ Franquès \\ 1, E-08028 Barcelona, Spain \\ ${ }^{2}$ Institute of Nanoscience and Nanotechnology (IN2UB), Universitat de Barcelona, Av. Joan XXIII S/N, \\ E-08028 Barcelona, Spain \\ ${ }^{3}$ Laboratory of Nanotechnology, Department of Microsystems Engineering (IMTEK), \\ Albert-Ludwigs-Universität Freiburg, Georges-Köhler-Allee 103, D-79110 Freiburg, Germany \\ ${ }^{4}$ CIMAP Normandie Univ, ENSICAEN, UNICAEN, CEA, CNRS, 14050 Caen, France \\ ${ }^{5}$ Nanophotonics Technology Center, Universitat Politècnica de València, Camino de Vera s/n, \\ E-46022 Valencia, Spain
}

(Received 3 July 2018; accepted 14 October 2018; published online 29 October 2018)

\begin{abstract}
The resistive switching properties of ITO/ZnO/p-Si devices have been studied, which present welldefined resistance states with more than five orders of magnitude difference in current. Both the high resistance state (HRS) and the low resistance state (LRS) were induced by either sweeping or pulsing the voltage, observing some differences in the HRS. Finally, the charge transport mechanisms dominating the pristine, HRS, and LRS states have been analyzed in depth, and the obtained structural parameters suggest a partial re-oxidation of the conductive nanofilaments and a reduction of the effective conductive area. Published by AIP Publishing. https://doi.org/10.1063/1.5046911
\end{abstract}

The poor energy efficiency of CMOS transistors and the fast scaling in memories are considered a serious drawback within the new era of big data and Internet of Things, which leads to the search of a new generation of ultra-low power nanodevices to overcome this important challenge that the electronics industry faces. Resistive switching (RS) memories or resistance random access memories (ReRAMs) have become a solution for the next generation of nonvolatile memories thanks to their low-power operation, high switching speed, and compatibility with the CMOS technology. ${ }^{1-4}$ In these devices, the switching between the low resistance state (LRS) and the high resistance state (HRS), when an external electric field is applied, is due to either ionic movement leading to atomic rearrangement inside the active layer (valence change mechanism, VCM) or metallic diffusion from the electrode (electro-chemical metallization, ECM), thus inducing the formation and destruction of conductive nanofilaments (CNFs). ${ }^{5,6}$ Different oxide compounds have been explored, such as $\mathrm{TiO}_{2}, \mathrm{HfO}_{2}, \mathrm{SnO}_{2}$, or $\mathrm{ZnO}$, which have demonstrated promising results. ${ }^{7-12}$ Among these metal oxides, $\mathrm{ZnO}$ has long attracted a great deal of attention because of its abundance and non-toxicity, giving rise to special interest also in gas sensing ${ }^{13,14}$ or as a transparent conductive oxide (TCO) in light-emitting and photovoltaic devices. Hence, this broad range of applications of $\mathrm{ZnO}$ makes it a potential candidate for the field of transparent ReRAM devices.

Here, we report on the RS properties of sputtered $\mathrm{ZnO}$ within an indium tin oxide (ITO)/ZnO/p-Si device configuration. The selection of ITO as the top contact also provides advantages over the control of the switching mechanisms, as no metal diffusion from the electrodes should occur. ${ }^{15-18}$ More than five orders of magnitude difference in current has been

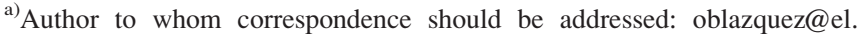
ub.edu
}

observed between LRS and HRS, with stable switching, taking place at low voltages $( \pm 1 \mathrm{~V})$ and with free current compliance. A cycling endurance beyond 1000 cycles has been demonstrated using pulse trains for performing the reading and the changes between the two resistance states. Finally, the obtained results are explained according to the charge transport mechanisms underlying the three different conduction states (pristine, LRS, and HRS), which sheds light on the CNF formation and destruction processes within $\mathrm{ZnO}$. Whilst other publications only perform a fitting of the experimental data using different conduction models but without discussing the extracted physical parameters, ${ }^{19-21}$ the hereby presented extended analysis of the $I(V)$ curves of our devices allowed for the determination of structural parameters related to RS, such as the effective conductive area in LRS and the filament gap in HRS. The determination of these parameters has previously been carried out directly by transmission electron microscopy (TEM) techniques. ${ }^{22-24}$ However, in-situ and ex-situ observation of the creation of those conductive paths and their interruption is a complex procedure, as well as the fact that sample preparation and/or the observation itself may modify the local structure.

Devices consisting of ITO/ZnO/p-type Si have been fabricated using a metal-oxide-semiconductor (MOS) configuration. For the oxide region, a 60-nm-thick layer was deposited on a $p$-type (100)-oriented silicon substrate by means of a 4-inch radiofrequency magnetron sputtering system from a pure $\mathrm{ZnO}$ target, using a power density value of $0.97 \mathrm{~W} \mathrm{~cm}^{-2}$, a $15 \mu$ bar Ar pressure, and a substrate temperature of $400^{\circ} \mathrm{C}$. An annealing process at $450^{\circ} \mathrm{C}$ for $1 \mathrm{~h}$ was carried out in a conventional furnace and under an Ar atmosphere. The top contact was achieved by deposition of ITO by electron-beam evaporation with subsequent annealing at $200^{\circ} \mathrm{C}$ in air for $1 \mathrm{~h}$. By using photolithography, devices with an effective area of $460 \times 460 \mu \mathrm{m}^{2}$ were fabricated. A sketch of the device structure is presented in Fig. 1(a) with the different materials that 

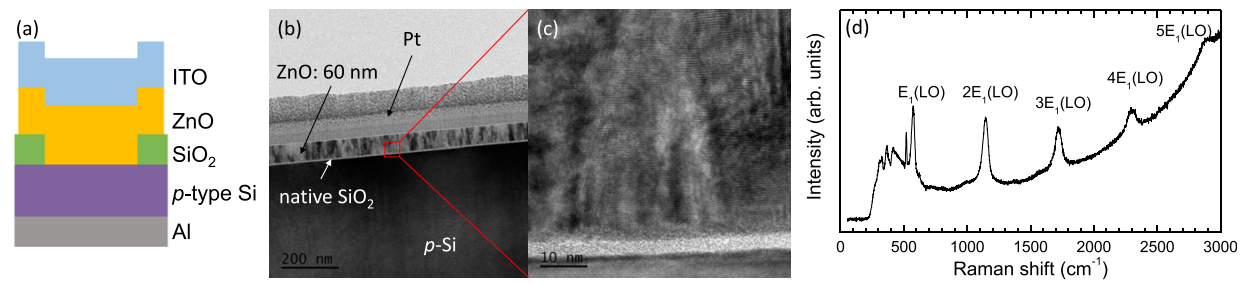

FIG. 1. (a) Sketch of the $\mathrm{ZnO}$-based devices under study in this work. (b) TEM image of the deposited $\mathrm{ZnO}$ active layer, where the thickness of the layer can be measured to be $\sim 60 \mathrm{~nm}$. (c) Zoom-in of the red-squared region in (b), where the polycrystalline nature of the layer can be identified. (d) Raman spectrum of a pristine device with the $\mathrm{E}_{1}(\mathrm{LO})$ vibrational mode of $\mathrm{ZnO}$ and its overtones up to the $5^{\text {th }}$ order, demonstrating its high crystalline quality.

compose each labelled layer. Energy-dispersive X-ray (EDX) analyses were performed on a $2-\mu$ m-thick $\mathrm{ZnO}$ film grown with the same conditions than those used for the active layer presented, by employing an OXFORD Instruments equipment monitored with the INCA software and installed on a JEOL 6400 SEM microscope operating at $20 \mathrm{kV}$. Raman measurements were done at room temperature using a Horiba Jobin Yvon LabRam spectrometer, exciting the sample with the 325$\mathrm{nm}$ line of a He-Cd laser. The electric characterization of the MOS devices was performed by means of an Agilent B1500 semiconductor device analyzer.

The $\mathrm{ZnO}$ active layer of the hereby presented resistive switching device has been characterized through diverse techniques. The TEM images of the $\mathrm{ZnO}$ layer are presented in Figs. 1(b) and 1(c). As it can be seen in Fig. 1(b), the $\mathrm{ZnO}$ active layer, deposited on top of the $\mathrm{Si}$ substrate, presents a thickness of $\sim 60 \mathrm{~nm}$. A native $\mathrm{SiO}_{2}$ thin layer can be seen at the interface with the Si substrate. Pt layers were deposited via focused ion beam (FIB) to prepare the sample for TEM. The polycrystalline nature of the $\mathrm{ZnO}$ active layer can be easily identified in Fig. 1(c). The composition of the $\mathrm{ZnO}$ active layer has been analyzed by means of EDX, resulting in 46 at.\% $\mathrm{O}$ and 54 at.\% $\mathrm{Zn}$, thus containing an expected oxygen deficiency due to the sputtering technique. A further analysis of the crystalline state of the $\mathrm{ZnO}$ layer has been carried out by means of Raman spectroscopy under near resonant conditions. In Fig. 1(d), it is displayed the spectra corresponding to a pristine device, where the $\mathrm{E}_{1}(\mathrm{LO})$ mode is shown up to the fifth order; this, together with a low value of the half-width at half-maximum of $\sim 13.7 \mathrm{~cm}^{-1}$ for the first peak, implies a high crystalline quality. ${ }^{25,26}$

The $I(V)$ characteristics of the devices were studied by applying a voltage on the top electrode while grounding the bottom contact, sweeping the voltage from $V=-1 \mathrm{~V}$ to $V$ $=+1 \mathrm{~V}$, thus performing RS cycles. In Fig. 2(a), we show a representative $I(V)$ curve out of more than 100 cycles, where the change from LRS to HRS is achieved at negative voltages, while the reverse change takes place at positive ones. Prior to it, an electroforming process was carried out by applying a positive ramp voltage and setting a current compliance at $5 \mathrm{~mA}$. The voltage at which this electroforming process takes place lies around $12.5 \mathrm{~V}$, leading the device to the LRS, as shown in the inset of Fig. 2(a). This state exhibits a much higher current than the pristine state, but without the need for using a limiting current compliance of the measuring system considering the explored range of voltages $( \pm 1 \mathrm{~V})$ : this state can thus be considered as a self-compliant LRS. The following step consists in applying a negative ramp voltage until
$-1 \mathrm{~V}$ in order to switch from LRS to HRS, leading to a considerable reduction in the current passing through the device, but being larger than the one observed in the pristine state. After applying another voltage sweep up to $1 \mathrm{~V}$, the LRS state is again recovered. In this cycle, the set and reset processes occur around $+0.4 \mathrm{~V}$ and $-0.4 \mathrm{~V}$, respectively.

At this point, the device was submitted to 100 consecutive self-compliant cycles by means of positive and negative ramp voltages in the range from $-1 \mathrm{~V}$ to $+1 \mathrm{~V}$ for set and reset steps, respectively [see the inset of Fig. 2(a)]. A current contrast of more than 5 orders of magnitude is observed between both states at a read voltage of $V_{\text {read }}=-0.2 \mathrm{~V}$ (LRS is plotted in blue, HRS in red). In order to analyze better these parameters along the 100 cycles, Fig. 2(b) shows the cumulative probability of the currents at $V_{\text {read }}$ for HRS and LRS. This plot confirms that both states are well defined and stable, with mean current values of $\sim 10^{-11} \mathrm{~A}$ and $\sim 2 \times 10^{-6}$
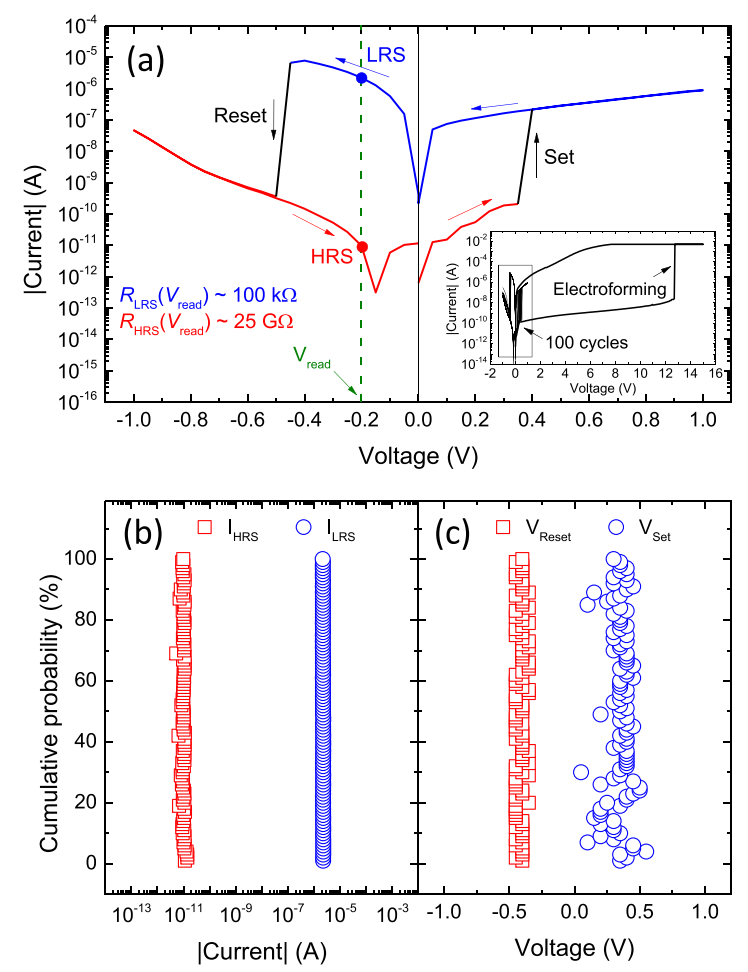

FIG. 2. (a) $I(V)$ characteristics of one RS cycle, where the voltage sweep direction of each cycle is indicated by arrows. HRS and LRS are colored in red and blue, respectively, whereas the set and reset processes are displayed in black. The vertical green dashed line indicates the read voltage $\left(V_{\text {read }}\right)$ where the current is analyzed. In the inset, the electroforming process is shown, followed by the 100 cycles, as a reference scale. (b) and (c) represent the cumulative probability of the HRS and LRS currents at $V_{\text {read }}$, and the cumulative probability of the $V_{\text {set }}$ and $V_{\text {reset }}$, respectively, averaged over the 100 cycles. 
A for HRS and LRS, respectively. The cumulative probabilities of the switching voltages between both states $\left(V_{\text {set }}\right.$ and $\left.V_{\text {reset }}\right)$ are shown in Fig. 2(c), which shows a clear variation between $V_{\text {set }}=+0.1 \mathrm{~V}$ and $+0.6 \mathrm{~V}$ (though always a positive voltage well below $1 \mathrm{~V}$ is required), whereas $V_{\text {reset }}$ presents a small variation always taking negative values between $-0.4 \mathrm{~V}$ and $-0.5 \mathrm{~V}$.

Taking into account the maximum $V_{\text {set }}$ and $V_{\text {reset }}$ values observed along the 100 cycles (in absolute value), the device was submitted to a specific pulse-voltage pattern, as shown in Fig. 3(a). In brief, the first pulse forces the set by applying $+2 \mathrm{~V}$, followed by a second pulse at $V_{\text {read }}=-0.2 \mathrm{~V}$ to read the current of the LRS. The third pulse, at $-1 \mathrm{~V}$, induces the reset towards HRS. Finally, the current at $V_{\text {read }}=-0.2 \mathrm{~V}$ is again monitored within this state. The duration of the set and reset pulses was kept at $50 \mathrm{~ms}$, long enough to promote switching, as observed experimentally; on the contrary, reading times were set to a much faster value, $0.1 \mathrm{~ms}$, limited by the experimental setup. The device presented an endurance of more than 1000 periods with $99.5 \%$ of success, whose cumulative probability is presented in Fig. 3(b). In this case, LRS presents the same current values than the observed ones when the device was submitted to voltage ramps via $I(V)$ curves [see Fig. 2(b)], taking values centered again around $\sim 2 \times 10^{-6} \mathrm{~A}$. This confirms that the LRS is well defined under both excitation conditions. In contrast, HRS presents a larger current than the one obtained using $I(V)$ curves. Whereas voltage ramps induced stable current intensity around $\sim 10^{-11} \mathrm{~A}$, pulse excitation increases the current intensity up to $\sim 10^{-9} \mathrm{~A}$. This difference in the current values within the HRS could be related to the short duration of the pulse during the reset process, which affects the atomic arrangement, as suggested by Marchewka et al. in $\mathrm{TaO}_{\mathrm{x}}$-based ReRAMs. ${ }^{27}$

In order to shed light on the physics underlying the memristive behavior of the $\mathrm{ZnO}$-based device under study, the charge transport mechanisms governing the different states (pristine, HRS and LRS) were determined in the substrate accumulation regime ( $V<0$ range). Figure 4(a) presents the isolated $I(V)$ curves corresponding to each of
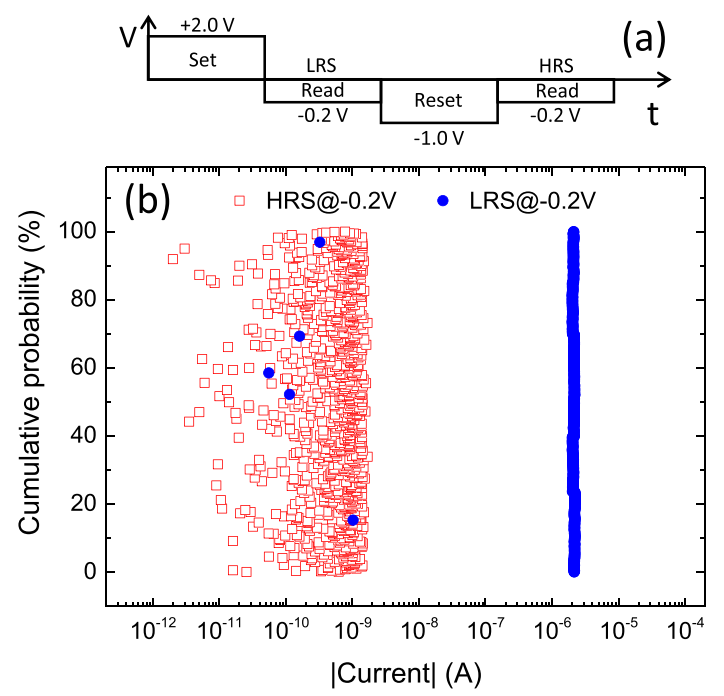

FIG. 3. (a) Scheme of the pulse-voltage pattern employed in the study. (b) Cumulative probability plot of HRS and LRS currents at $V_{\text {read }}$ under the pulse schematics in (a), after more than 1000 periods. these three states for the most representative cycle in Fig. 2(a), in the log-log representation. The three states present differentiated trends: the current intensity in the LRS exhibits a polynomial dependence on the applied voltage, whereas this dependence is exponential in the pristine state. In the HRS the situation is more complex and a combination of at least two mechanisms takes place.

In the pristine state, the trap-assisted tunneling (TAT) mechanism might presumably be the dominant conduction mechanism in our devices, as $\mathrm{ZnO}$ is an undoped material with intrinsic intra-band electronic states (due to its defective nature) $;^{28}$ a sketch of the energy band diagram for the TAT mechanism is depicted in Fig. 4(b). Under these conditions, the electrical current depends exponentially on the applied voltage, which is given by the following expression: ${ }^{28}$

$$
I=\frac{q n_{t} S}{2 \tau} \exp \left(-\frac{8 \pi \sqrt{2 m^{*}} d \phi_{t}^{3 / 2}}{3 h q V}\right),
$$

with $q$ being the elementary charge, $n_{\mathrm{t}}$ the trap areal density, $S$ the area of the device, $\tau$ the relaxation time between subsequent tunneling events, $m^{*}$ the effective mass of electrons, $d$ the thickness through which the electric field is applied, $\phi_{t}$ the offset energy between the electrode Fermi level and the trap level, and $h$ the Planck constant.

Regarding the LRS, the observed high current intensity suggests a large amount of injected carriers, which is compatible with the space charge-limited current (SCLC) theory [see Fig. 4(c)], exhibiting a quadratic dependence on the voltage $^{29}$

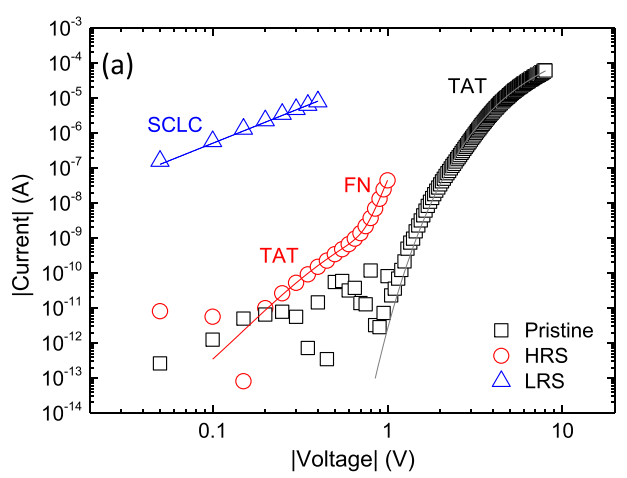

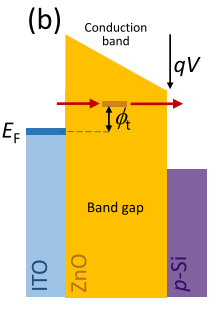

TAT

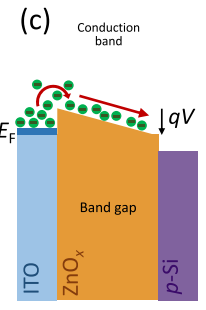

SCLC

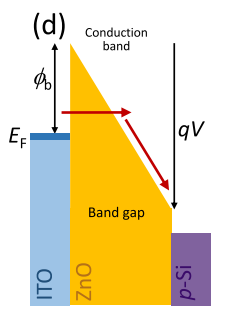

FN
FIG. 4. (a) Log-log representation of the $I(V)$ characteristics of pristine (black squares), HRS (red circles), and LRS (blue triangles) states. The fitting curves (in solid line) are also plotted together with the experimental data: trap-assisted tunneling (TAT) for pristine (in black), a combination of TAT and Fowler-Nordheim (FN) for HRS (in red) and space charge-limited current (SCLC) for LRS (in blue). All curves were acquired in substrate accumulation conditions $(V<0)$. Sketches of the energy band diagram of TAT, SCLC, and FN charge transport mechanisms are depicted in (b), (c), and (d), respectively. Whereas TAT and FN mechanisms take place in the $\mathrm{ZnO}$ (pristine and HRS), the SCLC mechanism occurs in $\mathrm{ZnO}_{\mathrm{x}}$ (LRS). 


$$
I=\frac{9}{8} \mu \varepsilon_{0} \varepsilon_{r} S \frac{V^{2}}{d^{3}},
$$

where $\mu$ is the electron drift mobility, and $\varepsilon_{0}$ and $\varepsilon_{\mathrm{r}}$ are the vacuum and relative permittivities, respectively. Finally, in the reset process, the conductivity of the devices dramatically decreases, reaching the HRS. Thus, the $\mathrm{ZnO}$ active layer becomes highly resistive and the intrinsic intra-band states are again relevant for the conduction. We observed that the current intensity dependence on the applied voltage exhibits two different trends, separated at a threshold voltage around $0.7 \mathrm{~V}$ [see Fig. 4(a)]. At voltages below this threshold, the TAT mechanism should again dominate charge transport, whereas Fowler-Nordheim $^{30}$ (FN) could be the main conduction mechanism at higher electric fields, due to the further lowering of the potential barrier. Therefore, a combination of both mechanisms can be considered as follows:

$$
\begin{aligned}
I= & \frac{q n_{t} S}{2 \tau} \exp \left(-\frac{8 \pi \sqrt{2 m^{*}} d \phi_{t}^{3 / 2}}{3 h q V}\right) \\
& +\frac{q^{2} S}{8 \pi^{2} h d^{2} \phi_{b}} V^{2} \exp \left(-\frac{8 \pi \sqrt{2 m^{*}} d \phi_{b}^{3 / 2}}{3 h q V}\right),
\end{aligned}
$$

where the first and second terms of the right side of the equation correspond to TAT and FN models, respectively; with $\phi_{b}$ being the energy offset between the ITO electrode Fermi level and the $\mathrm{ZnO}$ conduction band [see Figs. 4(b) and 4(d)].

Under these considerations, we have fitted the experimental $I(V)$ curves of the pristine, LRS and HRS states with the expressions from Eqs. (1), (2), and (3), respectively. This procedure allows us extracting physical parameters from the devices which are relevant to understand the RS mechanism within. Although obtaining these parameters is possible through other techniques, in-situ measurements often do not take into account sample damaging during preparation, whereas ex-situ ones can lead to many time-consuming attempts that do not often yield a specific result due to the difficulty of finding a CNF.

For the pristine state, we have fitted the experimental data using Eq. (1) taking into account that the current flows over the full device area through the 60-nm-thick $\mathrm{ZnO}$ layer, and using an electron effective mass for $\mathrm{ZnO}$ of $0.3 m_{\mathrm{e}},{ }^{31}$ which results in $\phi_{t}$ being around $0.20 \mathrm{eV}$. Considering this mechanism, the current is properly adjusted along more than six orders of magnitude, although some deviations are observed at voltages lower than $2 \mathrm{~V}$. In fact, some other effects such as charge trapping can occur and, thus, the parameters that could be obtained from the pre-exponential factor are subjected to a large error.

Using Eq. (2), we have fitted the experimental data from LRS, obtaining an excellent agreement with the space charge-limited current (SCLC) conduction mechanism. From the fit, and assuming that for a substoichiometric $\mathrm{ZnO}_{x}$ the electron drift mobility ranges between 18 and $140 \mathrm{~cm}^{2} \mathrm{~V}^{-1}$ $\mathrm{s}^{-1}$ and the relative permittivity is above $8.3,{ }^{32,33}$ we have found that the effective device area that is contributing to the conduction should be equal to or below $1.4 \times 10^{-10} \mathrm{~cm}^{2}$, much lower than the actual top ITO contact area $\left(2.1 \times 10^{-3}\right.$ $\mathrm{cm}^{2}$ ). This result suggests that the high current intensity observed in the LRS flows through filaments along the $\mathrm{ZnO}$ semiconductor layer. Taking into account the maximum active surface evidenced by the conduction mechanism, the current density along the CNFs can be evaluated, obtaining a lower limit of $J=1.6 \times 10^{4} \mathrm{~A} \mathrm{~cm}^{-2}$ at $V_{\text {read }}=-0.2 \mathrm{~V}$. This current density is indeed excessively high, but still lower than the one observed in the $\mathrm{TaO}_{x} / \mathrm{TiO}_{2} / \mathrm{TaO}_{x}$ structure, where values over $10^{7} \mathrm{~A} \mathrm{~cm}^{-2}$ were achieved. ${ }^{34}$ Further assuming that, in the set process, out-diffusion of oxygen ions is forming CNFs with diameters of about $25 \mathrm{~nm}$ (typical diameters reported in the literature lie between 10 and $50 \mathrm{~nm}),{ }^{11,12,35,36}$ the found active surface corresponds to 20 CNFs. Thus, this result suggests the formation of a limited number of conduction paths, with nanoscale dimensions.

Finally, we could adequately fit the HRS $I(V)$ curve to Eq. (3). Actually, the experimental current intensity for voltages below $V=0.7 \mathrm{~V}$ is properly reproduced by considering only the TAT contribution. The fact that the TAT mechanism is dominant at low voltages suggests conduction through the $\mathrm{ZnO}$ defective states, in a similar way than in the pristine state. This assumption points out to CNFs being interrupted, presumably due to their partial re-oxidation. The overall current through the $\mathrm{ZnO}$ layer is limited by this gap region, which contributes to the HRS. Assuming that the current flows through a $\mathrm{ZnO}$ layer with similar structural and chemical composition than the one in the pristine state $\left(\phi_{t}=0.20 \pm 0.02 \mathrm{eV}\right)$, we found an effective thickness reduction of about $85 \%$ (effective thickness of $9 \pm 1 \mathrm{~nm}$ ). The electric field along the gap of the interrupted CNFs is enhanced by, at least, this thickness variation, leading to a value of $\sim 0.8 \mathrm{MV} \mathrm{cm}{ }^{-1}$ at $V=0.7 \mathrm{~V}$ (see, for instance, Ref. 37, where there is an enhancement of the electric field in $\mathrm{Ga}: \mathrm{ZnO}$ nanofibers due to boundary effects). This electric field is enough to inject carriers with high kinetic energy to overcome the ITO-ZnO band offset $\left(\phi_{b}\right)$, making dominant the FN mechanism. Actually, the experimental data is well reproduced by the FN model, with a band offset energy of $\phi_{b}$ $=0.57 \pm 0.04 \mathrm{eV}$, which is below the energy that carriers have at voltages above $0.7 \mathrm{~V}$. The application of voltages larger than $1 \mathrm{~V}$ will produce again the set process, presumably by promoting out-diffusion of oxygen atoms towards the electrode.

The modeling of the pristine state, LRS and HRS in ZnO-based devices has allowed determining the mechanism responsible for the conduction in each of them, as well as important structural parameters. In the literature, these three states have also been analyzed: (i) the pristine shows trapassisted conduction typical of dielectric materials, like Poole-Frenkel or TAT, in good agreement with our observations. $^{38,39}$ On the other hand, (ii) the LRS typically exhibits a high conductance with an Ohmic or SCLC behavior; considering the effective areas reported so far, ${ }^{12}$ the current density reaches values compatible with $\mathrm{ZnO}$ with a high density of oxygen vacancies, indicating that the CNF formation is due to the out-diffusion of $\mathrm{O}$ atoms and/or oxygen deficiency in the film grown by magnetron sputtering. Finally, (iii) the HRS at low voltages presents again conduction typical of dielectric materials assisted by traps, suggesting that the conductive paths are partially re-oxidized, thus inducing the interruption of the CNFs along $9 \mathrm{~nm}$. At larger voltages (above $0.7 \mathrm{~V}$ ), injected carriers from the ITO contact present enough energy to overcome the ITO-ZnO potential barrier, exhibiting a FN behavior. ${ }^{19}$ The correlation between the 
charge transport mechanisms through $\mathrm{ZnO}$ and its structural modification under external electrical stress helps anticipating the optimum operation conditions of $\mathrm{ZnO}$-based memristors. In addition, subsequent cycles between positive and negative voltages can promote stable switching between the two states (HRS and LRS), maintaining the previously observed mechanisms.

The measurements and analyses have been extended to other cycles and devices with the same structure, finding similar results that lie within the error bars given for the extracted parameters. Further study on a stressed device and comparison with a pristine one (not shown) has provided prove of the modification of the $\mathrm{ZnO}$ structure, by comparing the resonant Raman $\mathrm{E}_{1}(\mathrm{LO})$ overtones between both devices. As it has previously been suggested, ${ }^{40,41}$ this fact is compatible with our hypothesis of oxygen out-diffusion in RS. Thus, the use of electrodes non-transparent to oxygen, ITO in our case, becomes an important factor in improving the endurance of these $\mathrm{ZnO}$-based devices.

Here, we have demonstrated the RS properties of ITO/ $\mathrm{ZnO} / p$-type $\mathrm{Si}$ devices. A difference in current of more than 5 orders of magnitude is observed between LRS and HRS, with endurance beyond $10^{3}$ cycles, while working at low voltages. The analysis of the intensity-voltage curves has shown the formation of CNFs at the set process due to out-diffusion of $\mathrm{O}$ atoms, which is responsible for the LRS. In the reset process, these CNFs are interrupted along $9 \mathrm{~nm}$ by their reoxidation, recovering the $\mathrm{ZnO}$ defective layer in this region. The fact that this region is in the range of some nanometers propitiated the observed high stability of the RS cycle in these devices. Overall, $\mathrm{ZnO}$, when combined with a $p$-type Si substrate and an ITO top electrode, is demonstrated as an excellent candidate for a future generation of RS memories, whose combination with other Si-based devices provides a large range of applications in transparent electronics.

This work was financially supported by the Spanish Ministry of Economy and Competitiveness (Project Nos. TEC2012-38540-C02-01 and TEC2016-76849-C2-1-R). O.B. also acknowledges the subprogram "Ayudas para Contratos Predoctorales para la Formación de Doctores" of the Spanish Ministry of Economy and Competitiveness for economical support. X.P., C.L., and C.G. are grateful to C. Frilay for his expertise in the maintenance of the sputtering kit used for the growth of the $\mathrm{ZnO}$ films.

${ }^{1}$ I. G. Baek, M. S. Lee, S. Sco, M. J. Lee, D. H. Seo, D.-S. Suh, J. C. Park, S. O. Park, H. S. Kim, I. K. Yoo, U.-I. Chung, and J. T. Moon, in IEDM Technical Digest. IEEE International Electron Devices Meeting, 2004 (IEEE, 2004), pp. 587-590.

${ }^{2}$ R. Waser and M. Aono, Nat. Mater. 6, 833 (2007).

${ }^{3}$ S. Kaeriyama, T. Sakamoto, H. Sunamura, M. Mizuno, H. Kawaura, T. Hasegawa, K. Terabe, T. Nakayama, and M. Aono, IEEE J. Solid-State Circuits 40, 168 (2005).

${ }^{4}$ D. B. Strukov and K. K. Likharev, Nanotechnology 16, 888 (2005).

${ }^{5}$ A. Mehonic, S. Cueff, M. Wojdak, S. Hudziak, O. Jambois, C. Labbé, B. Garrido, R. Rizk, and A. J. Kenyon, J. Appl. Phys. 111, 074507 (2012).

${ }^{6}$ A. Mehonic, A. Vrajitoarea, S. Cueff, S. Hudziak, H. Howe, C. Labbé, R. Rizk, M. Pepper, and A. J. Kenyon, Sci. Rep. 3, 2708 (2013).
${ }^{7}$ M. D. Pickett, G. Medeiros-Ribeiro, and R. S. Williams, Nat. Mater. 12, 114 (2013).

${ }^{8}$ S. H. Jo, T. Chang, I. Ebong, B. B. Bhadviya, P. Mazumder, and W. Lu, Nano Lett. 10, 1297 (2010).

${ }^{9}$ G. Vescio, A. Crespo-Yepes, D. Alonso, S. Claramunt, M. Porti, R. Rodriguez, A. Cornet, A. Cirera, M. Nafria, and X. Aymerich, IEEE Electron Device Lett. 38, 457 (2017).

${ }^{10}$ I. Valov, ChemElectroChem 1, 26 (2014).

${ }^{11}$ G. Martín, M. B. González, F. Campabadal, F. Peiró, A. Cornet, and S. Estradé, Appl. Phys. Express 11, 014101 (2018).

${ }^{12}$ F. M. Simanjuntak, D. Panda, K.-H. Wei, and T.-Y. Tseng, Nanoscale Res. Lett. 11, 368 (2016).

${ }^{13}$ J. Kim and K. Yong, J. Phys. Chem. C 115, 7218 (2011).

${ }^{14}$ Q. Yuan, Y. Zhao, L. Li, and T. Wang, J. Phys. Chem. C 113, 6107 (2009).

${ }^{15}$ J. W. Seo, J.-W. Park, K. S. Lim, J.-H. Yang, and S. J. Kang, Appl. Phys. Lett. 93, 223505 (2008).

${ }^{16}$ S. Z. Rahaman, S. Maikap, H.-C. Chiu, C.-H. Lin, T.-Y. Wu, Y.-S. Chen, P.-J. Tzeng, F. Chen, M.-J. Kao, and M.-J. Tsai, Electrochem. Solid-State Lett. 13, H159 (2010).

${ }^{17}$ F. M. Simanjuntak, D. Panda, T.-L. Tsai, C.-A. Lin, K.-H. Wei, and T.-Y. Tseng, J. Mater. Sci. 50, 6961 (2015).

${ }^{18}$ F. M. Simanjuntak, O. K. Prasad, D. Panda, C.-A. Lin, T.-L. Tsai, K.-H. Wei, and T.-Y. Tseng, Appl. Phys. Lett. 108, 183506 (2016).

${ }^{19}$ F. M. Simanjuntak, D. Panda, T.-L. Tsai, C.-A. Lin, K.-H. Wei, and T.-Y. Tseng, Appl. Phys. Lett. 107, 033505 (2015).

${ }^{20}$ Q. Liu, W. Guan, S. Long, R. Jia, M. Liu, and J. Chen, Appl. Phys. Lett. 92, 012117 (2008).

${ }^{21}$ Y. Shuai, S. Zhou, D. Bürger, M. Helm, and H. Schmidt, J. Appl. Phys. 109, 124117 (2011).

${ }^{22}$ J.-Y. Chen, C.-L. Hsin, C.-W. Huang, C.-H. Chiu, Y.-T. Huang, S.-J. Lin, W.-W. Wu, and L.-J. Chen, Nano Lett. 13, 3671 (2013).

${ }^{23}$ W. A. Hubbard, A. Kerelsky, G. Jasmin, E. R. White, J. Lodico, M. Mecklenburg, and B. C. Regan, Nano Lett. 15, 3983 (2015).

${ }^{24}$ Q. Liu, J. Sun, H. Lv, S. Long, K. Yin, N. Wan, Y. Li, L. Sun, and M. Liu, Adv. Mater. 24, 1844 (2012).

${ }^{25}$ X. Zhu, H.-Z. Wu, D.-J. Qiu, Z. Yuan, G. Jin, J. Kong, and W. Shen, Optics Commun. 283, 2695 (2010).

${ }^{26}$ M. F. Cerqueira, M. I. Vasilevskiy, F. Oliveira, A. G. Rolo, T. Viseu, J. Ayres de Campos, E. Alves, and R. Correia, J. Phys.: Condens. Matter 23, 334205 (2011).

${ }^{27}$ A. Marchewka, B. Roesgen, K. Skaja, H. Du, C.-L. Jia, J. Mayer, V. Rana, R. Waser, and S. Menzel, Adv. Electron. Mater. 2, 1500233 (2016).

${ }^{28}$ M. Krzywiecki, L. Grządziel, A. Sarfraz, D. Iqbal, A. Szwajca, and A. Erbe, Phys. Chem. Chem. Phys. 17, 10004 (2015).

${ }^{29}$ P. N. Murgatroyd, J. Phys. D: Appl. Phys. 3, 151 (1970).

${ }^{30}$ R. H. Fowler and L. Nordheim, Proc. R. Soc. A 119, 173 (1928).

${ }^{31}$ Ü. Özgür, Y. I. Alivov, C. Liu, A. Teke, M. A. Reshchikov, S. Doğan, V. Avrutin, S.-J. Cho, and H. Morkoç, J. Appl. Phys. 98, 041301 (2005).

${ }^{32}$ E. M. Kaidashev, M. Lorenz, H. von Wenckstern, A. Rahm, H.-C. Semmelhack, K.-H. Han, G. Benndorf, C. Bundesmann, H. Hochmuth, and M. Grundmann, Appl. Phys. Lett. 82, 3901 (2003).

${ }^{33}$ D. Gall, J. Appl. Phys. 119, 085101 (2016).

${ }^{34}$ W. Lee, J. Park, S. Kim, J. Woo, J. Shin, G. Choi, S. Park, D. Lee, E. Cha, B. H. Lee, and H. Hwang, ACS Nano 6, 8166 (2012).

${ }^{35}$ D.-H. Kwon, K. M. Kim, J. H. Jang, J. M. Jeon, M. H. Lee, G. H. Kim, X.S. Li, G.-S. Park, B. Lee, S. Han, M. Kim, and C. S. Hwang, Nat. Nanotechnol. 5, 148 (2010).

${ }^{36}$ B. J. Choi, A. C. Torrezan, J. P. Strachan, P. G. Kotula, A. J. Lohn, M. J. Marinella, Z. Li, R. S. Williams, and J. J. Yang, Adv. Funct. Mater. 26, 5290 (2016).

${ }^{37}$ X. Sun, SPIE Newsroom 82, 1096 (2006).

${ }^{38}$ C. Hu, Q. Wang, S. Bai, M. Xu, D. He, D. Lyu, and J. Qi, Appl. Phys. Lett. 110, 073501 (2017).

${ }^{39}$ F. Gul and H. Efeoglu, Superlattices Microstruct. 101, 172 (2017).

${ }^{40}$ O. Blázquez, G. Martín, I. Camps, A. Mariscal, J. López-Vidrier, J. M. Ramírez, S. Hernández, S. Estradé, F. Peiró, R. Serna, and B. Garrido, Nanotechnology 29, 235702 (2018).

${ }^{41}$ G. Bersuker, D. C. Gilmer, D. Veksler, P. Kirsch, L. Vandelli, A. Padovani, L. Larcher, K. McKenna, A. Shluger, V. Iglesias, M. Porti, and M. Nafría, J. Appl. Phys. 110, 124518 (2011). 